\title{
GÉNERO, SERVICIOS DE CUIDADOS Y ESTADO DE BIENESTAR: DESAFÍOS Y DEMANDAS EN TIEMPOS DE PANDEMIA MUNDIAL
}

\section{GENDER, CARE SERVICES AND THE WELFARE STATE: CHALLENGES AND DEMANDS IN TIMES OF A GLOBAL PANDEMIC}

\author{
LOURDES GABRIELA JimENEZ BRITO* \\ Conferencia Interamericana de Seguridad Social (CISS), México \\ lourdesjimenezb@hotmail.com
}

\section{RESUMEN:}

La pandemia mundial causada por el Covid-19 ha visibilizado la desigual carga de tareas reproductivas y domésticas que realizan las mujeres y la falta de sistemas públicos de cuidados universales y accesibles. La pandemia golpea de forma desproporcionada más a las mujeres ya que son ellas quienes ven aumentada exponencialmente la carga de tareas domésticas, en especial las vinculadas al cuidado. El actual escenario constituye una oportunidad histórica para demandar un nuevo pacto social que ponga a los cuidados en el centro del debate político, impulsando un renovado y fortalecido Estado de Bienestar con verdadera perspectiva de género y de derechos.

\section{Palabras clave:}

Pandemia mundial, igualdad de género, servicios de cuidados, seguridad social, Estado de Bienestar.

\section{ABSTRACT:}

The global pandemic caused by Covid-19 has made visible the unequal burden of reproductive and domestic tasks performed by women and the lack of universal

\footnotetext{
Candidata a Doctora en Ciencia Política por el Centro de Investigación y Docencia Económicas (CIDE).
} 
and accessible public care systems. The pandemic hits women more disproportionately as they are the ones who see the burden of homework on the domestic sphere exponentially increased, especially those linked to the care of others. The current scenario is a historic opportunity to demand a new social pact that puts care at the heart of the political debate, promoting a renewed and strengthened welfare state with a true perspective of gender and rights.

\section{Keywords:}

World pandemic, gender equality, care services, social security, Welfare State.

\section{INTRODUCCIÓN}

La Organización Mundial de la Salud (OMS) declaró, el once de marzo de 2019, que el brote de Covid-19 ha de considerarse como una pandemia mundial. ${ }^{1}$ Dicha pandemia ha puesto al mundo en crisis, por lo que no debe extrañarnos que también golpee al bienestar del núcleo familiar. La familia, como unidad básica de socialización y también como unidad económica, está siendo dramáticamente afectada por las consecuencias sociales del Covid-19. Millones de hogares alrededor del mundo están intentando sobrellevar un confinamiento o cuarentena dentro de sus casas, siguiendo recomendaciones de prevención dictadas por sus gobiernos con el fin de frenar la propagación del virus. ${ }^{2}$ Esta situación ha agudizado otra crisis que ya existía pero que no estaba siendo lo suficientemente visibilizada en el espacio público: la desigual carga de tareas reproductivas y domésticas que realizan las mujeres en el espacio privado y la falta de sistemas públicos de cuidados universales y accesibles. Entonces cabe preguntarse ¿Cómo afecta la actual pandemia sanitaria mundial a las mujeres? y además ¿Cómo debe responder el Estado ante la crisis de los sistemas de cuidados? Son numerosas las organizaciones y actores que han evidenciado como la actual pandemia golpea de forma desproporcionada y en mayor medida a las mujeres en general, y a las madres en particular, ya que son ellas quienes ven aumentada exponencialmente la carga de tareas en la esfera doméstica, en especial las vinculadas al cuidado de los otros. La situación desatada por el Covid-19 es una oportunidad histórica para demandar un nuevo pacto social que ponga a los cuidados y a todas las tareas del mundo reproductivo en el centro del debate político, impulsando un renovado y fortalecido Estado de Bienestar con verdadera perspectiva de género y de derechos.

Este artículo aborda esta problemática desde una doble perspectiva: una perspectiva histórica-cultural que identifica como la división sexual del trabajo es una construcción cultural que pone a las mujeres en una situación de inferioridad relegándolas al espacio doméstico y responsabilizándolas —casi exclusivamente - de las tareas reproductivas. Y en segundo lugar se hace un análisis

1 Alocución de apertura del Director General de la OMS en la rueda de prensa sobre la COVID-19 celebrada el 11 de marzo de 2020.

2 Área de especialistas de la Conferencia Interamericana de Seguridad Social, Maternidad y paternidad en tiempos del coronavirus SARS-COV-2, mayo 2020, disponible en https://ciss-bienestar.org/wp-content/ uploads/2020/05/maternidad-y-paternidad-en-tiempos-del-coronavirus-sars-cov-2.pdf 
desde la formulación de políticas públicas para poner en el centro del debate político la responsabilidad estatal de proveer servicios profesionales de cuidados y de promover políticas de igualdad de género en la materia. Uno de los desafíos planteados para el Estado a partir de esta pandemia mundial es redefinir su rol como proveedor de servicios y transferencias desde un esquema de seguridad social universal que compense - en un primer momento - los costos asumidos por las mujeres en el mundo reproductivo; pero que, en definitiva, promueva un verdadero esquema corresponsable de las tareas del mundo reproductivo, en donde múltiples actores — públicos y privados — gestionen adecuadamente dichas tareas para el bienestar de toda la sociedad.

El argumento central de este trabajo es que la pandemia desatada el Covid-19 ha tenido como una de sus consecuencias más importantes la visibilización de la necesidad de redefinir una nueva estrategia sociopolítica de cuidados que permita a toda la población ejercer su derecho a ser cuidados y a cuidar. Esto implica una política de concertación entre actores públicos y privados que gestione adecuadamente la organización social de los cuidados. En este sentido, sostengo que, frente a este nuevo escenario, el Estado debe llevar la rectoría de tal estrategia y responder con políticas públicas con perspectiva de género que garanticen igualdad para las mujeres respecto a los hombres en relación con la rígida división sexual del trabajo vigente en nuestras sociedades. La reproducción biológica, pero también la crianza de los nuevos miembros de la sociedad, son tareas fundamentales que permiten la realización de la vida tal cual la conocemos. Los beneficios y las utilidades que crea son tan grandes y difíciles de cuantificar que no podríamos equiparar este trabajo con ningún otro. Las tareas de cuidados y crianza al haber sido elegidas histórica y culturalmente como trabajo de mujeres, ha llevado que las madres en particular, pero las mujeres en general sostengan con su trabajo no remunerado el mundo reproductivo, situándolas en clara desventaja respecto de los hombres. Paradójicamente, el mundo productivo - y remunerado- no sería posible sin esa carga de trabajo a cargo de las mujeres. Ellas no solo gestan y dan a luz con su cuerpo, sino que también, alimentan, cuidan, visten, limpian y educan casi en forma permanente. ${ }^{3}$

La estructura del presente artículo es la siguiente: en primer lugar, analizamos desde una perspectiva histórico-cultural como la división sexual de tareas reproductivas y productivas constituye el origen de la desigualdad entre hombres y mujeres. A continuación, se hace referencia al derecho a cuidar y ser cuidado que tenemos todas las personas. En la siguiente sección se aporta evidencia con datos en base a las denominadas encuestas de uso del tiempo a partir de las cuales es posible dimensionar la cantidad de trabajo reproductivo no remunerado que hacen las mujeres en la esfera doméstica, comparado con la carga menor que asumen los hombres. Como consecuencia de esta desigual carga de trabajo, las mujeres disponen de menos tiempo para dedicarse al mundo productivo y por lo tanto

3 JIMENEZ BRITO, Lourdes Gabriela, Maternidad y Seguridad Social, Conferencia Interamericana de Seguridad Social, 2020. Inédito al momento de citarse. 
remunerado. Seguidamente el trabajo se enfoca en las principales razones que pueden explicar cómo y por qué la pandemia del Covid-19 afecta desproporcionadamente más a las mujeres que a los hombres. Finalmente se aborda la necesidad de redefinir un nuevo pacto social que ponga a las tareas reproductivas en el centro del debate político. Es urgente politizar la cuestión de los cuidados y demandar que el Estado sea el principal proveedor de políticas con pretensión de universalidad a partir de un principio de igualdad de género para que sus ciudadanos puedan ejercer su derecho a cuidar y ser cuidados.

\section{EL ORIGEN DE LA DESIGUALDAD: LA RÍGIDA DIVISIÓN SEXUAL DEL TRABAJO}

El trabajo reproductivo hace referencia al trabajo destinado a satisfacer las necesidades de la familia. A pesar de constituir una dimensión necesaria para la reproducción de la sociedad, su desarrollo ha quedado históricamente circunscrito al marco privado, primordialmente a la esfera doméstica. ${ }^{4}$ Incluye actividades de cuidados, crianza, alimentación, vestimenta, mantenimiento de los espacios domésticos, entre otras múltiples tareas que permiten la supervivencia de la vida. En este sentido, no debería haber dudas acerca de que la reproducción social debe ser una preocupación central del Estado, ya que a través de ella se garantiza la subsistencia misma de la sociedad. Una primera aproximación conceptual de lo que se entiende como "reproducción social" es la de Johanna Brenner y Barbara Laslett (1991) quienes la definen como:

[...] las actividades, actitudes, comportamientos, emociones, responsabilidades y relaciones directamente involucradas en el mantenimiento de la vida, en forma diaria e intergeneracionalmente. Implica varios tipos de trabajo socialmente necesario (mental, físico y emocional) destinado a proporcionar los medios definidos histórica, social, así como biológicamente, para mantener y reproducir la población. Entre otras cosas, la reproducción social incluye cómo la comida, la ropa y el refugio están disponibles para el consumo inmediato, cómo se realiza el mantenimiento y la socialización de los niños, cómo se proporciona el cuidado de los ancianos y los enfermos, y cómo se construye socialmente la sexualidad. ${ }^{5}$

Sin estas actividades reproductivas esenciales, el mundo productivo no sería posible. El gran problema es que estas tareas están subvaloradas y mayormente desprotegidas. Históricamente, este trabajo de reproducción social ha sido elegido como trabajo de mujeres, y a menudo sin paga o protección. Sin él no podría haber cultura, economía ni organización política. De hecho, ninguna sociedad que socava sistemáticamente la reproducción social puede perdurar por mucho tiempo. ${ }^{6}$

4 LARRAÑAGA, Isabel; ARREGI, Begoña y ARPAL, Jesús «El trabajo reproductivo o doméstico», Gac Sanit 18, núm 1 (2004): 31-37.

5 BRENNER, Johanna y LASLETT, Barbara «Gender, Social Reproduction, and Women's Self-Organization: Considering the U.S. Welfare State», Gender and Society 5, núm 3 (1991): 311-33.

6 FRASER, Nancy «Crisis of Care? On the Social Reproductive Contradictions of Contemporary Capitalism», en Social Reproduction Theory Remapping Class, Recentering Oppression (London: Pluto Press, 2017), 21. 
La dedicación femenina al trabajo de la reproducción es el resultado de la construcción social de las diferencias de género. Se trató, sin lugar a duda, de una construcción histórica y cultural respecto a lo que significa la feminidad y la masculinidad. Esa construcción convierte a las hembras en sujetos femeninos, orientados centralmente hacia el trabajo de la reproducción y a los machos, en sujetos masculinos, centrados básicamente en el trabajo de la producción. Tal proceso de socialización condiciona las posibilidades materiales de vida, las actitudes y las representaciones simbólicas de ambos géneros y posiciona a las mujeres en situación de subordinación respecto a los hombres. Es en esa socialización diferencial de género que se consigue que esas diferencias devengan desigualdades y que, además, éstas permanezcan invisibles. ${ }^{7}$ Por lo tanto, la reproducción social se sostiene en gran medida gracias a la instauración de esta idea injusta que responsabiliza a las mujeres de todas esas tareas: desde los aspectos fisiológicos que supone la maternidad (gestación, parto y lactancia) pero también todas aquellas vinculadas a la crianza, los cuidados y el mantenimiento de la vida que nada tienen que ver con la biología. Esa es la denuncia de Lagarde, quien sostiene que vivimos en una sociedad que especializa a las mujeres en la reproducción social, quienes, a su vez, lo han internalizado como parte de sí mismas $\mathrm{y}$ este rol constituye un núcleo fundamental de la identidad femenina. ${ }^{8}$

Las orientaciones de lo público y lo privado, igual que las del género, se arraigan en lo que los antropólogos llaman habitus: las convenciones por las cuales experimentamos, como si fuera natural, nuestros propios cuerpos y movimientos en el espacio del mundo. ${ }^{9}$ Destaca Warner que, en el caso del género, lo público y lo privado no son sólo reglas formales sobre cómo deben comportarse los hombres y las mujeres; sino que, además, están cargadas de significados de masculinidad y feminidad. La masculinidad, al menos en las culturas occidentales, se siente en parte en una manera de ocupar el espacio público; la feminidad, en un lenguaje de sentimiento privado. ${ }^{10}$ Lo que se quiere manifestar es que la vida privada, cotidiana, emerge como el espacio de la reproducción social, de la maternidad, de los cuidados y asuntos domésticos. Es razonable argumentar que este habitus, es decir, estas convenciones acerca de los roles que deben asumir las mujeres y los hombres se ve alimentado desde dos vertientes: el Estado que, a través de ciertas políticas públicas, alimenta y cristaliza ciertos estereotipos de

7 CARRASQUER, Pilar et. al., «El trabajo reproductivo», Papers. Revista de Sociologia 55 (1 de julio de 1998): 97, https://doi.org/10.5565/rev/papers.1934

8 Para señalar este destino casi obligatorio de todas las mujeres, Lagarde creó la denominada categoría de "madresposa": [...] el objeto sobre el que se aplica el trabajo de la madresposa es el ser humano. Su trabajo y, de manera más amplia, sus actividades vitales, consisten en reproducir materialmente, en su corporeidad, al otro, pero también consisten, desde el nacimiento y en los primeros años de vida, en humanizar al ser humano en su propia cultura, de acuerdo con su género, clase, grupo y tradiciones [...] La mujer valora más la existencia del otro que la propia, porque sólo su reconocimiento le da existencia a ella misma. LAGARDE DE LOS RÍOS, Marcela, Los cautiverios de las mujeres: Madresposas, monjas, putas, presas y locas (Siglo XXI Editores México, 2016).

9 WARNER, Michael Público, públicos, contrapúblicos, trad. Victoria Schussheim, $1^{\circ}$ (México: Fondo de Cultura Económica, 2012), 20.

10 Idem. 
género y, la sociedad en general que especializa a las mujeres en las tareas de la reproducción social.

A partir de estas consideraciones es posible identificar cómo todas aquellas funciones propias de la reproducción social han sido, histórica e injustamente, asignadas a las mujeres casi en su totalidad. Es cierto que la capacidad fisiológica de la mujer para dar vida constituye un hecho biológico y natural. Sin embargo, todas las otras dimensiones de la maternidad y demás funciones que implican la reproducción social, no tienen por qué ser ejercidas o asumidas en su totalidad por las mujeres, o en su caso, por las madres. Los argumentos religiosos, científicos y naturalistas buscaban convencer a las mujeres para que dieran prioridad a la crianza frente a otros aspectos de su vida. Las madres fueron relegadas a la esfera privada, con el objetivo de cuidar de la "prole", mientras se las apartaba del ámbito público. Así fue el proceso de "maternalización de la mujer" cuando la condición de madre pasó a ser la única identidad posible, una identidad exclusiva y excluyente. ${ }^{11}$

Cuando las mujeres asumen completamente las tareas reproductivas, disponen de menos tiempo para dedicarse a las tareas productivas y remuneradas. Es preciso desestructurar a las mujeres como "seres para los otros", como los entes maternos, y socializar los cuidados que prodigan: maternizar a la sociedad y des maternizar a las mujeres. Para ello es prioritario distribuir los cuidados vitales de los otros, cuando menos paritariamente entre la maternidad, la paternidad y la ampliación y creación de instituciones sociales públicas que los realicen. ${ }^{12}$

\section{CUIDAR Y SER CUIDADO: ESENCIAL PARA LA VIDA}

El cuidado es concebido actualmente como una responsabilidad primaria de las familias que debe resolverse en el ámbito privado. ${ }^{13}$ La consideración del cuidado como un derecho universal, en su potestad de reclamar el derecho a ser cuidado, a cuidar y a cuidarse (autocuidado), significa que no se trata solo de impulsar acciones que aumentan la oferta de servicios reproductivos (educativos, de primera infancia, salud, culturales, seguridad social), fundamentales sin duda, sino que transversalmente se aborden las responsabilidades, permisos legales, arreglos familiares y societales: inversión pero también reconocimiento. ${ }^{14}$ Además, la conceptualización del cuidado como derecho conlleva para el Estado, naturalmente, no sólo obligaciones negativas (abstenerse de entorpecer los servicios de guarderías infantiles, no impedir el acceso de un adulto mayor al sistema de salud) sino también obligaciones positivas, tales como proveer los medios para poder cuidar y garantizar que el cuidado se lleve adelante en condiciones de igualdad. ${ }^{15}$

11 VIVAS, Esther, Mamá desobediente (Capitán Swing, 2019).

12 LAGARDE DE LOS RÍOS, Marcela Los cautiverios de las mujeres, p. 824.

13 GHERARDI, Natalia y ZIBECCHI, Carla, «El derecho al cuidado: ¿Una nueva cuestión social ante los tribunales de justicia de Argentina?», Política. Revista de Ciencia Política 49, núm 1 (12 de octubre de 2011): 107-138.

14 PAUTASSI, Laura «Del "boom" del cuidado al ejercicio de derechos», Revista Internacional de Derechos Humanos, Conectas Derechos Humanos, 13, núm. 24 (2016): 35-42.

15 PAUTASSI, Laura, El cuidado como cuestión social desde un enfoque de derechos, CEPAL (Santiago de Chile: CEPAL, 2007), https://www.cepal.org/es/publicaciones/5809-cuidado-como-cuestion-social-un-enfoque-derechos. 
Cabe destacar que el trabajo de cuidar a los demás, ya sea remunerado o no, a menudo implica más conexión personal, apego emocional y compromiso moral que otras formas de trabajo. Crea beneficios tanto públicos como privados y su valor es difícil de medir. ${ }^{16}$ Todos estos factores ponen a los proveedores de cuidados en desventaja económica. No debe de sorprender que, incluso en el ámbito del trabajo remunerado fuera del hogar, las mujeres sigan desempeñando un rol vinculado a los cuidados. La mayoría de las mujeres latinoamericanas todavía están empleadas en el comercio (30\%), educación y salud (23\%) y servicios domésticos (11\%). La participación de estos sectores representa más del 60\% del empleo de mujeres en total; y esa participación no ha cambiado sustancialmente en los últimos veinte años. ${ }^{17}$ Folbre argumenta que la especialización en cuidados se mantiene porque las mujeres no pueden amenazar fácilmente con retirar sus servicios, no pueden medir fácilmente el valor de sus contribuciones y no pueden reclamar personalmente todo el valor que crean. ${ }^{18}$

El trabajo de cuidar a las personas dependientes crea efectos secundarios positivos para la sociedad en su conjunto, creando y manteniendo la próxima generación de trabajadores y ciudadanos. Sin embargo, los individuos que pagan los costos más altos en términos de tiempo y dinero obtienen pocos beneficios pecuniarios. Tanto las empresas capitalistas como las empresas públicas tienden a dar por hecho la mayor oferta de capital humano y social. Recompensan y promueven a las personas que se dedican al trabajo remunerado, centrándose en resultados que se miden fácilmente en la métrica del mercado. Como resultado, los empleados que se comprometen mucho tiempo con el trabajo familiar o comunitario generalmente pagan una gran multa social y económica. ${ }^{19}$ En definitiva, la carga del cuidado sí tiene un precio y no es inusual que sea mayormente una mujer quien deje la fuerza laboral o reduzca sus horas o aspiraciones profesionales para cumplir con dichas tareas, contribuyendo así a las brechas de género en el empleo y las ganancias.

Por otra parte, la evolución del Estado de Bienestar refleja importantes cambios históricos en la relación entre la familia y la economía. Sin embargo, las políticas públicas familiares no han podido seguir el ritmo de los cambios económicos y demográficos. Una oferta pública más generosa de cuidado de niños y

16 El trabajo doméstico y de cuidados que tiene lugar en las familias de forma no remunerada equivale a entre $15 \%$ y $25 \%$ de todo lo que se produce en una sociedad. El grueso de ese trabajo lo hacen las mujeres. Por cada punto del PIB que invierte el Estado, las mujeres aportan una contraparte no monetaria. Por ejemplo: si tienes una escuela pública, alguien tiene que llevar a la niña a la escuela, buscarla, traerla, hacer las tareas con ella y apoyarla. Pensar los cuidados en medio de la gran pandemia, entrevista a MARTíNEZ FRANZONI, Juliana, Nueva Sociedad, mayo 2020. Disponible en https://nuso.org/articulo/pensar-los-cuidados-enmedio-de-la-gran-pandemia/

17 MARCHIONNI, Mariana «A changing scenario: education, family and economic environment», en Bridging gender gaps? The rise and deceleration of female labor force participation in Latin America (Universidad Nacional de La Plata, 2015).

18 FOLBRE, Nancy, "The Care Penalty and Gender Inequality", en Susan Averett et. al., (eds.), The Oxford Handbook of Women and the Economy, Oxford University Press, New York, 2018.

19 BITTMAN, Michael y Folbre, Nancy Family Time: The Social Organization of Care: 2 (London; New York, 2004). 
licencia familiar remunerada, así como mejores salarios y beneficios para el empleo a tiempo parcial, podrían ayudar a los trabajadores a encontrar un mejor equilibrio. ${ }^{20}$ No obstante, la pandemia mundial por Covid-19 ha puesto al mundo reproductivo de cabeza y exige soluciones innovadoras, inclusivas y universales que permitan a las familias ejercer su derecho a cuidar y ser cuidados en forma efectiva.

La necesidad de un modelo de organización social que coloque los cuidados en el centro los valore, los haga visibles y señale que son responsabilidad de todos, con una imprescindible implicación del Estado, es imperiosa. Ser cuidado y cuidar es un derecho y un deber en una sociedad que sitúe en un lugar prioritario la vulnerabilidad de la vida. ${ }^{21}$ Reconocer la importancia del cuidado y de la reproducción social no sólo tiene una dimensión académica, sino también política. Una redistribución más justa del cuidado implica romper la lógica mercantil, establecer una nueva alianza con la seguridad social, fortalecer las redes de apoyo comunitarias y conseguir una participación equitativa de mujeres y hombres en el cuidado. ${ }^{22}$

Ezquerra Samper propone, por ejemplo, que el trabajo de cuidados, en términos de justicia de género, debe plantearse desde una doble perspectiva: el reparto entre hombres y mujeres y la socialización más allá del núcleo familiar. ${ }^{23}$ Por otra parte, Vivas plantea organizar los cuidados y el empleo a partir del modelo de "cuidador universal", es decir, personas que combinen empleo y cuidados, rompiendo así la separación tradicional entre el rol de proveedor y el de cuidador. El reto consiste en pensar un tipo de organización social que lo haga posible. Una sociedad que acoja el cuidado y la maternidad en plenitud solo será posible si revaloriza dichas tareas, las reparte de forma igualitaria entre hombres y mujeres, las socializa reforzando los servicios públicos y las iniciativas comunitarias, reduce drásticamente la jornada laboral y, más en general, organiza la vida pública de manera permeable a las necesidades de las personas. ${ }^{24}$

\section{ENCUESTAS DE USO DE TIEMPO: EVIDENCIA DE LA DIVISIÓN SEXUAL DEL TRABAJO IMPERANTE}

Una herramienta de gran utilidad para conocer la real división de tareas entre hombres y mujeres son las encuestas sobre uso del tiempo. Se trata de la única herramienta disponible para medir el trabajo de cuidados no remunerado y también es un método más rentable para recopilar datos oportunos y precisos sobre la división del trabajo por género dentro de los hogares y la interdependencia del trabajo remunerado y no remunerado realizado por mujeres y hombres. ${ }^{25}$ Cuando no se realizan encuestas de uso de tiempo, esto constituye una omisión

20 Idem.

21 VIVAS, Esther, Mamá desobediente, op. cit., p. 118.

22 COMAS D'ARGEMIR, Dolors, La crisis de los cuidados: ¿la “otra” crisis?, 9 de abril de 2014, El Diario.es

23 EZQUERRA SAMPER, Sandra «¿Qué hacer con los cuidados: De la Economía Feminista a la democratización de los cuidados», Viento sur: Por una izquierda alternativa, No 156 (2018): 39-47.

24 VIVAS, Esther, Mamá desobediente, op. cit.

25 STUART, Sheila «Situation of Unpaid Work and Gender in the Caribbean: The Measurement of Unpaid Work through Time-Use Studies», enero de 2014, https://repositorio.cepal.org//handle/11362/36619 
grave porque significa que el trabajo no remunerado, en particular el trabajo de cuidados no remunerado, a pesar de su importante contribución al desarrollo económico y social, no se refleja en las estadísticas económicas utilizadas para la formulación de políticas, a saber, las cuentas nacionales y las estadísticas oficiales del mercado laboral. ${ }^{26}$

Las mujeres, en particular las que son madres, dedican comparativamente mucho más tiempo a las tareas domésticas y de cuidado no remunerados que los hombres. Esto supone un costo de oportunidad muy grande para ellas y una disminución del tiempo que podrían dedicar a actividades productivas remuneradas. Según un estudio elaborado por la Organización para la Cooperación y el Desarrollo Económicos (OCDE) titulado Equilibrio entre trabajo remunerado, trabajo no remunerado y ocio del año 2018, encuentra que el tiempo dedicado al trabajo no remunerado expone enormes brechas de género. Las mujeres mexicanas pasan la mayor parte del tiempo en estas tareas, contabilizando hasta 6 horas y 23 minutos al día, mientras que los hombres mexicanos dedican un promedio de 2 horas y 17 minutos al trabajo no remunerado. Luego le siguen las mujeres portuguesas que pasan 5 horas y 28 minutos al día en trabajo no remunerado, mientras que los varones portugueses pasan solo 1 hora y 36 minutos, la cifra más baja en Europa después de Turquía. ${ }^{27}$ A medida que la tasa de participación de la mujer en la fuerza laboral se aproxima a la de los hombres y su nivel educativo aumenta, Averett, Argys y Hoffman evidencian una reducción de la diferencia en el tiempo que las esposas y los esposos dedican al trabajo doméstico y al cuidado de los hijos. ${ }^{28}$

Es interesante observar que las mujeres han aumentado el tiempo que dedican al trabajo remunerado de manera mucho más espectacular que los hombres han aumentado la cantidad de tiempo que dedican al cuidado familiar. Y es que el ejercicio de la masculinidad en el ámbito doméstico ha cambiado muy poco. Eso no quiere decir que no haya hombres que lo hagan, sino que el ejercicio de las masculinidades en relación con el trabajo doméstico y de cuidados no ha cambiado lo suficiente para reflejarse en las estadísticas. El uso del tiempo masculino en el hogar es el recurso más democráticamente repartido en toda la región. Mientras que las mujeres muestran una profunda desigualdad entre ellas, asociadas a los niveles de educación formal y a los niveles de ingresos (cuanto mayores son, menos horas se emplean en el trabajo doméstico y de cuidados no remunerado), entre los hombres hay una enorme homogeneidad. Se requiere una intervención deliberada del Estado y de otros actores de la sociedad, para lograr un mayor involucramiento de los hombres en las tareas reproductivas. Se necesitan mensajes más claros y fuertes que reconcilien masculinidad y cuidado. ${ }^{29}$ A continuación los datos sobre el uso del tiempo de mujeres y hombres para América Latina.

\footnotetext{
26 Idem.

27 Informe disponible en https://www.oecd.org/gender/balancing-paid-work-unpaid-work-and-leisure.htm

28 AVERETT, Argys, y HOFFMAN, op. cit., p. 20.

29 Nueva Sociedad Pensar los cuidados en medio de la gran pandemia, entrevista a Martínez Franzoni, Juliana, mayo 2020. Disponible en https://nuso.org/articulo/pensar-los-cuidados-en-medio-de-la-gran-pandemia/
} 


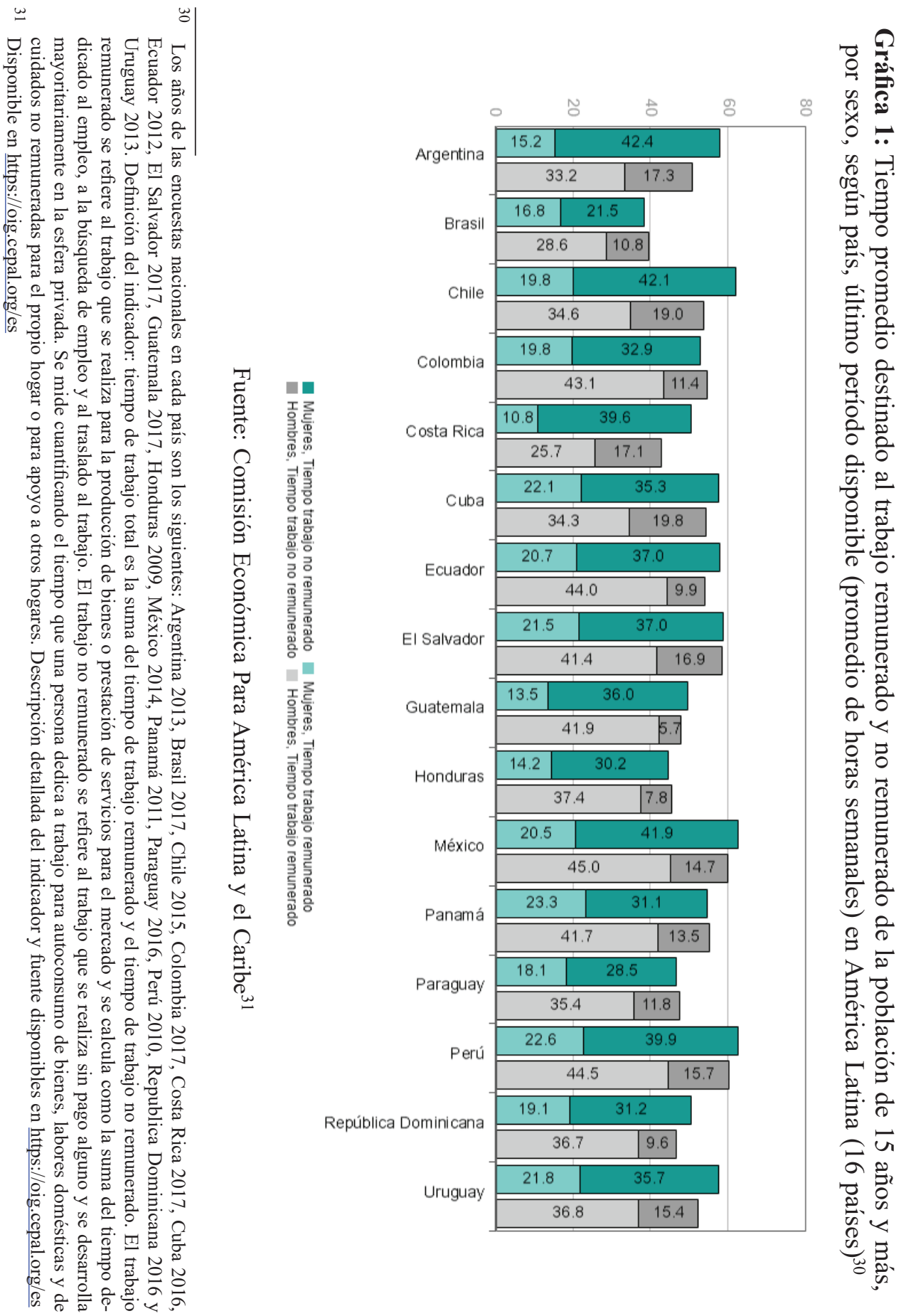


La gráfica 1 permite observar como las mujeres de la región latinoamericana dedican comparativamente mayor tiempo a las tareas no remuneradas, respecto a los hombres cada semana. Países como Chile, México y Perú representan los casos más agudos de brecha de género en donde las mujeres dedican más del doble de tiempo a tareas remuneradas. Y es que, en las sociedades latinoamericanas, las madres suelen ser las principales cuidadoras de los niños, lo que compromete su capacidad de participar activamente en actividades laborales. La evidencia es consistente con esta afirmación: en promedio, mientras que el 67 por ciento de las mujeres mayores de edad sin hijos menores de 18 años participan en el mercado laboral, esa proporción cae al 56 por ciento para aquellas con al menos un hijo menor de 5 años. ${ }^{32}$ Por lo tanto, es posible afirmar que la reducción de la carga de tiempo asociada a las responsabilidades familiares puede mejorar el empoderamiento económico de las mujeres. Y es que las decisiones importantes sobre educación, trabajo y fertilidad se superponen con la edad económica activa de las mujeres. Como resultado, las decisiones familiares pueden desplazar la inserción laboral de madres y esposas. ${ }^{33}$

Las mujeres, de este modo, tienen una "doble presencia" constante en la esfera productiva y reproductiva. ${ }^{34}$ Por el contrario, el trabajo remunerado proporciona a las mujeres un estatus por derecho propio, les otorga un mayor poder e influencia en la toma de decisiones dentro de la familia y eleva el nivel de vida de la familia en general. ${ }^{35}$ La sobrecarga del trabajo de cuidados no remunerado y la pobreza de tiempo de las mujeres impiden la igualdad de oportunidades, derechos y resultados frente a los hombres, en relación con la participación no sólo en el mercado laboral, sino también en la participación social y política y en el disfrute de tiempo libre. En definitiva, la sobrecarga de este tipo de trabajo sobre las mujeres limita sus oportunidades y se erige como un obstáculo para su empoderamiento económico y para el disfrute de sus derechos en igualdad de condiciones con los hombres. ${ }^{36}$

\section{PANDEMIA MUNDIAL: AGUDIZACIÓN DE LA CRISIS DEL MUNDO REPRODUCTIVO}

La crisis provocada por la pandemia del Covid-19 ha puesto de manifiesto la centralidad del trabajo de cuidados. Un trabajo que en los sistemas de salud y en los hogares permiten salvar vidas y sostener los hogares convertidos en el

$\overline{32}$ MARCHIONNI, Mariana, op. cit., p. 117.

33 GASPARINI, Leonardo y MARCHIONNI, Mariana, "Overview”, en Gasparini et.al. Bridging gender gaps? The rise and deceleration of female labor force participation in Latin America, Universidad Nacional de La Plata, Buenos Aires, 2015, p. 31.

34 BALBO, Laura, "La doble presencia", en Carmen Alemany et. al (eds.), Las mujeres y el trabajo: rupturas conceptuales, Icaria, Madrid, 1994, pp. 503-514.

35 BLAU, Francine D y WINKLER, Anne D. Eds.., «Gender Differences Around the World», in The Economics of Women, Men, and Work, Eighth edition (Oxford University Press, 2017), 463.

36 CEPAL y ONU Mujeres, Cuidados en América Latina y el Caribe en tiempos de COVID-19: hacia sistemas integrales para fortalecer la respuesta y la recuperación (CEPAL, 2020), 3, disponible en https://www.cepal. org/es/publicaciones/45916-cuidados-america-latina-caribe-tiempos-Covid-19-sistemas-integrales-fortalecer 
centro neurálgico de las medidas de confinamiento. Sin embargo, la crisis también ha demostrado la insostenibilidad de la actual organización social de los cuidados intensificando las desigualdades económicas y de género existentes, puesto que son las mujeres más pobres quienes más carga de cuidados soportan y a quienes, la sobrecarga de cuidados condiciona, en mayor medida, sus oportunidades de conseguir sus medios para la subsistencia. ${ }^{37}$

El Covid-19, como toda pandemia, ha desajustado los modelos de vida a los que estábamos acostumbrados. Esta dificultad sanitaria puso en evidencia que todos y todas somos interdependientes. La rápida propagación del virus y las medidas institucionales para producir aislamiento social que se están aplicando en la mayoría de los países, visibiliza uno de los eslabones más débiles de nuestra sociedad: los cuidados. ${ }^{38}$ Ante la situación actual, no se debe perder de vista que los impactos e implicaciones son diferentes para mujeres y hombres. Zamudio Santos encuentra que las mujeres son las más expuestas a los efectos adversos de los desastres. ${ }^{39}$ La principal razón de que a las mujeres les cuesta más recuperarse es porque cuentan con menos recursos económicos y financieros. Los estudios sobre las pandemias de ébola y zika han demostrado, por ejemplo, que las respuestas ante las crisis ignoraron las desigualdades estructurales entre los sexos, con lo que se exacerbaron los efectos negativos para las mujeres. ${ }^{40}$ Paradójicamente, las mujeres desempeñan un papel crucial en la recuperación de dichas contingencias, pues realizan la mayor parte de los trabajos no remunerados esenciales para las tareas de reproducción social. Son imprescindibles en la lucha contra el brote como trabajadoras y profesionales sanitarias, voluntarias comunitarias y cuidadoras. ${ }^{41}$

Cabe destacar que, ante el cierre de escuelas y centros de cuidados de menores y personas de la tercera edad, son mayormente las mujeres quienes deben permanecer en casa como responsables de los cuidados de estos grupos de riesgo. La expectativa social de que las mujeres atenderán a los enfermos limita su capacidad de controlar su riesgo de infección. ${ }^{42}$ El segundo aspecto para tener en cuenta es que las cuarentenas afectan fuertemente a la actividad económica, tanto formal

37 CEPAL y ONU Mujeres, Cuidados en América Latina y el Caribe en tiempos de Covid-19.

38 BATTHYÁNY, Karina, La pandemia evidencia y potencia la crisis de los cuidados, Consejo Latinoamericano de Ciencias Sociales, disponible en https://www.clacso.org/la-pandemia-evidencia-y-potencia-la-crisisde-los-cuidados/

39 ZAMUDIO SANTOS, Verhonica, Mujeres y niñas, las más afectadas por desastres., Investigación CISS, marzo 2020. Disponible en https://ciss-bienestar.org/wp-content/uploads/2020/03/ mujeres_ninas_las_mas_ afectadas_por_desastres.pdf

40 ELIES, Stefanie, La crisis de la Covid-19 y la vía hacia un nuevo paradigma, democraciaAbierta (dA), disponible en https://www.opendemocracy.net/es/la-crisis-de-la-Covid-19-y-la-v\%C3\%ADa-hacia-un-nuevoparadigma/

41 ONU Mujeres, Covid-19 en América Latina y el Caribe: cómo incorporar a las mujeres y la igualdad de género en la gestión de la respuesta a la crisis. Brief 1.1. 17.03.2020, disponible en https:/lac.unwomenúmrg/ es/digiteca/publicaciones/2020/03/covid-como-incorporar-a-lasmujeres-y-la-igualdad-de-genero-en-la-gestionde-respuesta

42 Measure Evaluation, The Importance of Gender in Emerging Infectious Diseases Data, 2017. Disponible en https://www.measureevaluationúmrg/resources/publications/fs-17-205a 
como informal. Las mujeres suelen ser quienes conforman, mayoritariamente, el mercado informal de trabajo. Por lo tanto, esta situación de contingencia por el brote de Covid-19 afecta en mayor medida a las mujeres tanto porque se las penaliza con una mayor carga de tareas de cuidados y por afectar directamente sus medios económicos de subsistencia. ${ }^{43}$

\section{HACÍA UNA ESTRATEGIA SOCIOPOLÍTICA DE CUIDADOS: POLÍTICAS PÚBLICAS EN TIEMPO DE PANDEMIA}

Tal como se ha venido sosteniendo, es preciso considerar a los cuidados dentro de la esfera pública, estatal y no solo privada. Siguiendo con esta lógica, se requiere consolidar la idea de que los cuidados son también responsabilidad de la sociedad en general y no solo de las familias y definitivamente no solo de las mujeres. El desafío político es poder poner en debate la incorporación de los cuidados como parte de un esquema de seguridad social de acceso universal. Y es que la interdependencia de las personas es un hecho de la vida en común, hoy agudizado por la pandemia de Covid-19. La solución no pasa solamente por repartir más equitativamente el cuidado entre varones y mujeres a nivel individual, sino que su importancia y valor se reconozca y pueda ser provisto también en parte por la sociedad y con el Estado asumiendo su responsabilidad. La única respuesta total y efectiva ante las crisis en la reproducción de la vida está dada por las instituciones universales, públicas y gratuitas, por los espacios de lo común, de lo colectivo. En esta situación de alarma, los Estados en general han convocado a la responsabilidad individual para hacer frente a una crisis estructural que evidencia las fragilidades de los sistemas públicos de atención a personas dependientes. ${ }^{44}$

Son varias las áreas de oportunidad que nos plantea la presente crisis sanitaria global: en primer lugar, demandar un mayor involucramiento de los varones en el trabajo doméstico no remunerado para que éste no recaiga injustamente de forma desproporcionada en las mujeres. En este sentido, se habla de la necesidad de reconocer, redistribuir y reducir el trabajo de cuidados no remunerado asumido por las mujeres para permitir que la responsabilidad sobre este trabajo fundamental sea valorada y asumida entre el Estado, el sector privado, la comunidad, los hogares y entre hombres y mujeres. ${ }^{45}$ De hecho un reciente estudio conducido por Harvard Graduate School of Education encuentra que el 68 por ciento de los padres se sienten más cerca o mucho más cerca de sus hijos desde la pandemia. ${ }^{46}$

43 Área de especialistas, Conferencia Interamericana de Seguridad Social, El brote de Covid-19 y su efecto desproporcionado en las mujeres, marzo de 2020, disponible en https://ciss-bienestar.org/wp-content/ uploads/2020/03/el_brote_de_covid_19_y_su_efecto_desproporcionado_en_las_mujeres_pdf

44 BATTHYÁNY, Karina La pandemia evidencia y potencia la crisis de los cuidados, Consejo Latinoamericano de Ciencias Sociales, https:/www.clacso.org/la-pandemia-evidencia-y-potencia-la-crisis-de-los-cuidados/ ELSON, Diane, «Recognize, Reduce, and Redistribute Unpaid Care Work: How to Close the Gender Gap», New Labor Forum 26, núm 2 (1 de mayo de 2017): 52-61, https://doi.org/10.1177/1095796017700135

46

Weissbourd, Richard, Batanova, Milena, McIntyre, Joseph, and Torres, Eric, How the Pandemic is Strengthening Fathers' Relationships with Their Children, June 2020, Making Caring Common Project Harvard 
Esto puede ser alentador. Sin embargo, tal como ha mencionado, el mayor involucramiento de los varones en las tareas reproductivas no sucederá por generación espontánea sino más bien como resultado de políticas y campañas activas que promuevan esquemas de corresponsabilidad de las tareas reproductivas.

La segunda área de oportunidad es debatir el diseño una estrategia pública de gestión de los cuidados que, junto a la provisión privada — familiar y la que se obtiene en el mercado laboral - constituyan una verdadera respuesta a la crisis que se vive en esta dimensión. Y es que los cuidados son una gran oportunidad para dinamizar la economía y para apostar a una economía distinta, centrada en las personas, pero hay que considerarlos como un trabajo valioso, visibilizarlo, remunerarlo y protegerlo. ${ }^{47}$ En esta sentido, la construcción de sistemas integrales de cuidados, a la par que un factor fundamental para el logro del empoderamiento, la autonomía de las mujeres y la igualdad de género, constituye un elemento clave de la recuperación socioeconómica en este escenario de crisis global por varias razones: (a) la inversión en infraestructura social de cuidados y la creación de una economía de servicios de cuidados, dinamiza las economías locales a partir de la creación directa de empleo y la mejora de los ingresos familiares; (b) contribuyen a la profesionalización y certificación de quienes trabajan en forma remunerada en este sector, aumentando sus capacidades laborales, sus salarios y su productividad y (c) la regulación y formalización del sector contribuye a la generación de ingresos tributarios y al fortalecimiento de los sistemas de seguridad social, en contextos donde la alta informalidad y la pérdida masiva de empleos formales pone en riesgo la sostenibilidad de estos sistemas. ${ }^{48}$

El recrudecimiento de la crisis de los cuidados como efecto de la pandemia no se resolverá haciendo pequeños y tímidos arreglos de política social. ${ }^{49} \mathrm{Al}$ contrario, el nuevo escenario exige un cambio radical en la forma de concebir las tareas reproductivas con el objetivo de implementar un modelo de seguridad social universal que incluya esta dimensión o pilar fundamental como son los cuidados de los otros. En términos de política pública, se busca acotar el concepto, considerándose políticas de cuidado aquellas acciones públicas orientadas a la organización del trabajo de atención y asistencia de las personas en situación de dependencia - para que puedan realizar las actividades básicas, instrumentales y avanzadas de la vida diaria - siendo también las personas que cuidan sujetos de estas políticas. Bajo este concepto se incluyen medidas que garanticen el acceso a servicios, tiempo y recursos para que las personas puedan cuidar y ser cuidadas

Graduate School of Education, disponible en https://static1.squarespace.com/static/5b7c56e255b02c683659fe43/ $\mathrm{t} / 5$ eeceba88f50eb19810153d4/1592585165850/Report+How+the+Pandemic + is + Strengthening+Fathers + Rel ationships+with+Their+Children+FINAL.pdf

ARAYA, Mabel y ZAGAL, Geanina, Crisis de cuidados en tiempos de pandemia, 18 de marzo de 2020, https://www.elmostrador.cl/braga/2020/03/18/crisis-de-cuidados-en-tiempos-de-pandemia/ cuidados en tiempos de pandemia», AraInfo · Diario Libre d'Aragón (blog), 30 de mayo de 2020, https:// arainfo.org/nos-deben-una-vida-reflexiones-sobre-la-crisis-de-los-cuidados-en-tiempos-de-pandemia/ 
en condiciones de calidad e igualdad. ${ }^{50}$ La Comisión Económica Para América Latina y el Caribe (CEPAL) recomienda que las políticas de cuidado deben sostenerse sobre la base de los principios de universalidad, solidaridad, autonomía y corresponsabilidad social, es decir, con intervención de las familias, el Estado, el mercado y la sociedad, y corresponsabilidad en términos de género entre hombres y mujeres.

Resulta importante destacar que varios países de la región han avanzado en un importante número de políticas y de acciones en desarrollo vinculadas al reconocimiento, reducción y redistribución de los cuidados. Los mayores avances se han dado en la creación de servicios de cuidado a la primera infancia. En otras áreas estratégicas, como la atención a personas adultas mayores dependientes y personas enfermas y con discapacidad, la regulación de licencias maternales y parentales para el cuidado, el fortalecimiento y formalización del sector del cuidado, los incentivos a organizaciones laborales flexibles y compatibles con las responsabilidades del cuidado, o la corresponsabilidad de los hombres con estas tareas, presentan un desarrollo más limitado e incipiente. ${ }^{51}$ Sin embargo, estas reformas se han dado en una etapa previa al inicio de la pandemia sanitaria actual. Esto lleva a considerar la necesidad de rediseñar estrategias novedades acordes a las demandas aquí planteadas.

\section{CONCLUSIONES}

A lo largo de este documento, se ha manifestado que la pandemia sanitaria global causada por el Covid-19 ha tenido como una consecuencia no intencionada visibilizar la importancia y centralidad para la vida misma de las tareas reproductivas, en particular las de cuidados de los otros. Los sistemas tradicionales de cuidados ya estaban en crisis dado que se fundamentaban en la concepción injusta e indigna de que las mujeres eran las responsables de tales trabajos. Sin embargo, la llegada de una pandemia mundial cuya medida más extendida para enfrentarla fue el confinamiento ciudadano en el espacio doméstico, exacerbó aún más la crisis de los cuidados. Las mujeres no pueden seguir sosteniendo con su trabajo no remunerado en la esfera privada, el mundo productivo. El actual escenario exige poner en el centro del debate político y social el diseño de una estrategia transversal de gestión de los cuidados a lo largo de todo el ciclo de la vida.

A modo de cierre, recapitulo las tres ideas fuertes que conforman el argumento de este documento: la rígida división sexual del trabajo vigente es el origen de la desigualdad entre hombres y mujeres ya que, a partir de ella, las mujeres disponen de menos tiempo para dedicarse al mundo productivo y por lo tanto remunerado. La evidencia a nivel agregado es consistente con este argumento pues los datos demuestran que las mujeres dedican más del doble de su tiempo a las

50 CEPAL y ONU Mujeres, Cuidados en América Latina y el Caribe en tiempos de Covid-19.

51 SALLÉ MARÍA, Ángeles y MOLPECERES, Laura, Reconocer, redistribuir y reducir el trabajo de cuidados. Prácticas inspiradoras en América latina y el Caribe. (ONU Mujeres, 2018), https://lac.unwomenúmrg// digiteca/publicaciones/2018/11/estudio-reconocer-redistribuir-y-reducir-el-trabajo-de-cuidados. 
tareas reproductivas en la esfera doméstica, en comparación con los hombres. Finalmente se analizó como la actual pandemia golpea en forma diferenciada a hombres y mujeres, agudizando aún más las brechas de género preexistentes. Y es que, con las medidas de confinamiento social, el cierre masivo de establecimientos educativos y de cuidados, el descalabro económico y la centralidad de las tareas sanitarias (donde hay una alta tasa de feminización), las mujeres terminan siendo las más afectadas por la actual crisis. Finalmente, se puso en discusión la necesidad de configurar la actual crisis como una ventana de oportunidad para demandar por parte del Estado una reconfiguración de su rol como proveedor de servicios de cuidados desde un esquema de seguridad social universal y con perspectiva de género. La extrema vulnerabilidad de la vida humana ha quedado en evidencia. Los Estados alrededor del mundo deberían responder con políticas públicas que valoricen, protejan y remuneren a las tareas reproductivas en general, pero a las tareas de cuidados en particular.

\section{BIBLIOGRAFÍA}

ARAYA, Mabel y ZAGAL, Geanina, Crisis de cuidados en tiempos de pandemia, 18 de marzo de 2020. Disponible en https://www.elmostrador.cl/braga/2020/03/18/crisis-de-cuidados-en-tiempos-de-pandemia/

AVERETT, Susan L.; ARGYS, Laura M. y HOFFMAN, Saul D., Eds. The Oxford Handbook of Women and the Economy. New York, NY: Oxford University Press, 2018.

BALBO, Laura. «La doble presencia». En Las mujeres y el trabajo: rupturas conceptuales, 1994, pp. 503-514, 503-14. Icaria, 1994.

BATTHYÁNY, Karina, La pandemia evidencia y potencia la crisis de los cuidados, Consejo Latinoamericano de Ciencias Sociales, disponible en https:// www.clacso.org/la-pandemia-evidencia-y-potencia-la-crisis-de-los-cuidados/

BITTMAN, Michael, y FOLBRE, Nancy. Family Time: The Social Organization of Care: 2. London; New York, 2004.

BRENNER, Johanna, y LASLETT, Barbara. «Gender, Social Reproduction, and Women's Self-Organization: Considering the U.S. Welfare State». Gender and Society 5, núm 3 (1991): 311-33.

CARRASQUER, Pilar; TORNS, Teresa; TEJERO, Elisabet, y ROMERO, Alfonso. «El trabajo reproductivo». Papers. Revista de Sociologia 55 (1 de julio de 1998): 95. https://doi.org/10.5565/rev/papers.1934

CEPAL, y ONU Mujeres. Cuidados en América Latina y el Caribe en tiempos de COVID-19: hacia sistemas integrales para fortalecer la respuesta y la recuperación. CEPAL, 2020. https://www.cepal.org/es/publicaciones/45916-cuidados-america-latina-caribe-tiempos-Covid-19-sistemas-integrales-fortalecer.

Conferencia Interamericana de Seguridad Social El brote de Covid-19 y su efecto desproporcionado en las mujeres, marzo de 2020, disponible en https://cissbienestar.org/wp-content/uploads/2020/03/el_brote_de_covid_19_y_su_efecto_desproporcionado_en_las_mujeres_.pdf 
Conferencia Interamericana de Seguridad Social, Maternidad y paternidad en tiempos del coronavirus Sars-CoV-2, mayo 2020, disponible en https:/cissbienestar.org/wp-content/uploads/2020/05/maternidad-y-paternidad-en-tiempos-del-coronavirus-sars-cov-2.pdf

Dolors Comas d'Argemir, La crisis de los cuidados: ¿la "otra" crisis?, 9 de abril de 2014, E1 Diario.es.

ELIES, Stefanie, La crisis de la Covid-19 y la vía hacia un nuevo paradigma, democraciaAbierta (dA). Disponible en https://www.opendemocracy.net/es/ la-crisis-de-la-Covid-19-y-la-v\%C3\%ADa-hacia-un-nuevo-paradigma/

ELSON, Diane. «Recognize, Reduce, and Redistribute Unpaid Care Work: How to Close the Gender Gap». New Labor Forum 26, núm 2 (1 de mayo de 2017): 52-61. https://doi.org/10.1177/1095796017700135.

EZQUERRA SAMPER, Sandra. « ¿Qué hacer con los cuidados? De la Economía Feminista a la democratización de los cuidados»Viento sur: Por una izquierda alternativa, núm 156 (2018): 39-47.

FOLBRE, Nancy. «The Care Penalty and Gender Inequality». The Oxford Handbook of Women and the Economy, 26 de julio de 2018.

FRANCINE D., Blau y WINKLER, Anne E., eds. «Gender Differences Around the World». En The Economics of Women, Men, and Work, Eighth edition, 560. Oxford University Press, 2017.

FRASER, Nancy. «Crisis of Care? On the Social Reproductive Contradictions of Contemporary Capitalism». En Social Reproduction Theory Remapping Class, Recentering Oppression. London: Pluto Press, 2017.

GASPARINI, Leonardo, y MARCHIONNI, Mariana. «Overview». En Bridging gender gaps? The rise and deceleration of female labor force participation in Latin America. Universidad Nacional de La Plata, 2015.

GHERARDI, Natalia, y ZIBECCHI, Carla. «El derecho al cuidado: ¿Una nueva cuestión social ante los tribunales de justicia de Argentina?» Política. Revista de Ciencia Politica 49, núm 1 (12 de octubre de 2011): 107/138-107/138. https://doi.org/10.5354/rp.v49i1.16742.

JIMENEZ BRITO, Lourdes Gabriela, Maternidad y Seguridad Social, Conferencia Interamericana de Seguridad Social, 2020. Inédito al momento de citarse.

JIMÉNEZ CASTILLÓN, Sofía y MORENO, Esther «Nos deben una vida: reflexiones sobre la crisis de los cuidados en tiempos de pandemia», AraInfo Diario Libre d'Aragón (blog), 30 de mayo de 2020. Disponible en https:// arainfo.org/nos-deben-una-vida-reflexiones-sobre-la-crisis-de-los-cuidadosen-tiempos-de-pandemia/.

LAGARDE DE LOS RÍOS, Marcela. Los cautiverios de las mujeres: Madresposas, monjas, putas, presas y locas. Siglo XXI Editores México, 2016.

LARRAÑAGA, Isabel; ARREGI, Begoña, y ARPAL, Jesús. «El trabajo reproductivo o doméstico». Gac Sanit 18, núm 1 (2004): 31-37.

Measure Evaluation, The Importance of Gender in Emerging Infectious Diseases Data, 2017. Disponible en https://www.measureevaluationúmrg/resources/publications/fs-17-205a 
MARCHIONNI, Mariana, "A changing scenario: education, family and economic environment». En Bridging gender gaps? The rise and deceleration of female labor force participation in Latin America. Universidad Nacional de La Plata, 2015.

Nueva Sociedad, Pensar los cuidados en medio de la gran pandemia, entrevista a Juliana Martínez Franzoni, mayo 2020. Disponible en https://nuso.org/articulo/pensar-los-cuidados-en-medio-de-la-gran-pandemia/

ONU Mujeres, Covid-19 en América Latina y el Caribe: cómo incorporar a las mujeres y la igualdad de género en la gestión de la respuesta a la crisis. Brief 1.1. 17.03.2020. Disponible en https://lac.unwomenúmrg/es/digiteca/publicaciones/2020/03/covid-como-incorporar-a-lasmujeres-y-la-igualdad-de-genero-en-la-gestion-de-respuesta

OCDE, Balancing paid work, unpaid work and leisure, 2018. Disponible en https:// www.oecd.org/gender/balancing-paid-work-unpaid-work-and-leisure.htm

PAUTASSI, Laura, El cuidado como cuestión social desde un enfoque de derechos. CEPAL. Santiago de Chile: CEPAL, 2007. https://www.cepal.org/es/ publicaciones/5809-cuidado-como-cuestion-social-un-enfoque-derechos.

PAUTASSI, Laura, «Del "boom” del cuidado al ejercicio de derechos». Revista Internacional de Derechos Humanos, Conectas Derechos Humanos, 13, núm 24 (2016): 35-42.

SALLÉ, María Ángeles y MOLPECERES, Laura, Reconocer, redistribuir y reducir el trabajo de cuidados. Prácticas inspiradoras en América latina y el Caribe. ONU Mujeres, 2018. https://lac.unwomenúmrg//digiteca/publicaciones/2018/11/estudio-reconocer-redistribuir-y-reducir-el-trabajo-de-cuidados.

STUART, Sheila. «Situation of Unpaid Work and Gender in the Caribbean: The Measurement of Unpaid Work through Time-Use Studies», enero de 2014. https://repositorio.cepal.org//handle/11362/36619.

VIVAS, Esther. Mamá desobediente. Capitán Swing, 2019.

WARNER, Michael. Público, públicos, contrapúblicos. Traducido por Victoria Schussheim. $1^{\circ}$. México: Fondo de Cultura Económica, 2012.

WEISSBOURD, Richard; BATANOVA, Milena; MCINTYRE, Joseph and TORRES, Eric, How the Pandemic is Strengthening Fathers' Relationships with Their Children, June 2020, Making Caring Common Project Harvard Graduate School of Education. Disponible en https://static1.squarespace.com/static/5b7c56e255b02c683659fe43/t/5eeceba88f50eb1981015 $3 \mathrm{~d} 4 / 1592585165850 /$ Report + How + the + Pandemic + is + Strengthening+Fathers + Relationships+with+Their+Children+FINAL.pdf

ZAMUDIO SANTOS, Verhonica, Mujeres y niñas, las más afectadas por desastres., Investigación CISS, marzo 2020. Disponible en https://ciss-bienestar. org/wp-content/uploads/2020/03/ mujeres_ninas_las_mas_afectadas_por_desastres.pdf 